\title{
Volumetric plasmonic resonators for very thin organic solar cells
}

\author{
Mustafa Akın Sefünç ${ }^{1}$, Ali Kemal Okyay ${ }^{1}$ and Hilmi Volkan Demir ${ }^{1,2 *}$ \\ ${ }^{1}$ Bilkent University, Department of Electrical and Electronics Engineering, Department of Physics, UNAM - National Nanotechnology \\ Research Center, and Institute of Materials Science and Nanotechnology, Bilkent, TR-06800, Ankara, Turkey \\ ${ }^{2}$ Nanyang Technological University, School of Electrical and Electronic Engineering, Division of Microelectronics, School of Physical \\ and Mathematical Sciences, Division of Physics and Applied Physics, Nanyang Avenue, Singapore 639798, Singapore \\ *Tel:[+90](312) 290 1021, e-mail: volkan@bilkent.edu.tr
}

New-generation thin-film solar cells are designed to feature very thin layers of active material in the order of tens of nanometers, which conveniently offers the advantage of cost reduction. However, this kind of architectures undesirably suffers limited total optical absorption of incident photons in these active layers. To address this problem, there has been an increasing interest in designing plasmonic structures around the active layers for enhancing their total optical absorption. Using a single layer of such plasmonic structures either on the top or at the bottom of these absorbing layers has been extensively studied in the literature ${ }^{1,2,3}$. In this work, different than the previous reports, we focus on a new design concept of volumetric plasmonic resonators that relies on the idea of coupling two (or more) layers of coupled plasmonic structures embedded in the organic solar cells. For this, here we incorporate one silver grating on the top of the absorbing layer and another at the bottom of the active layer in order to couple them with each other such that field localization is further increased within the volume of the active material between gratings. In addition to individual plasmonic resonances of these metallic structures, this allows us to take the advantage of the vertical interaction in the volumetric resonator. This interaction contributes to further enhancement of optical absorption in the active layer, beyond the limited photon absorption in non-metallic (bare) organic solar cell. Our results show that this architecture exhibits a substantial absorption enhancement performance particularly under the transverse magnetic (TM) polarized illumination, while the optical absorption is maintained at a similar under the transverse electric (TE) polarized illumination. As a result, the optical absorption in the active layer is enhanced up to $\sim 67 \%$.
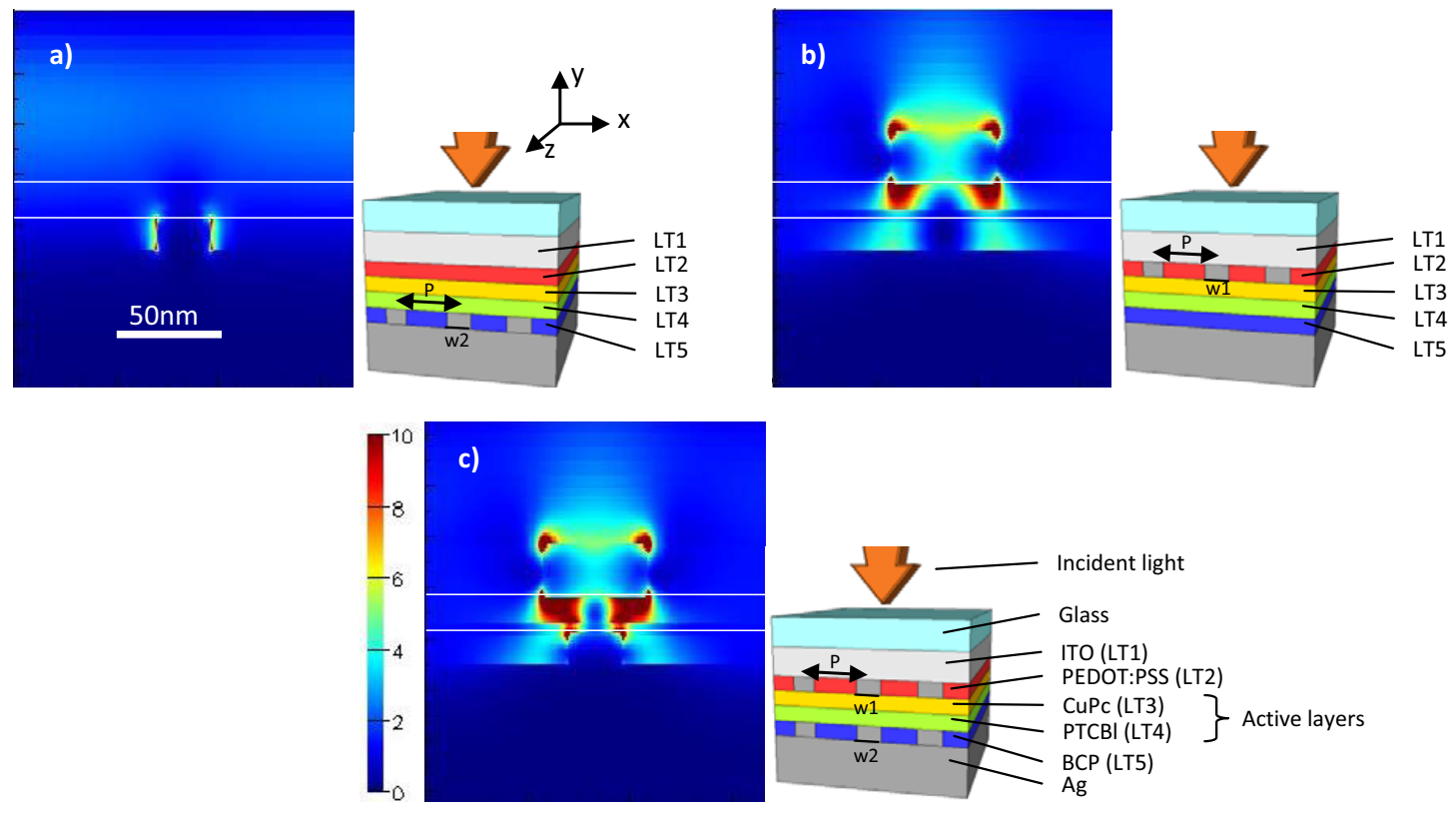

Fig. 1: Cross-sectional views of simulated solar cells incorporating plasmonic structures: Normalized electric field profiles for (a) only bottom silver grating,(b) only top silver grating, and (c) volumetric plasmonic resonators (including both top and bottom silver gratings), with the design parameters of $\mathrm{P}=200 \mathrm{~nm}$ (period of the grating), w1 $=50 \mathrm{~nm}$ (width of the top metal grating), and w $2=30 \mathrm{~nm}$ (width of the bottom metal grating), under TM-polarized normal-incident illumination at $\lambda=510 \mathrm{~nm}$. Next to electric field maps are shown the corresponding solar cell structures of ITO/PEDOT:PSS/CuPc/PTCB1/BCP/Ag, all of them (a)-(c) using exactly the same layer thicknesses of LT1=150 nm (ITO), LT2=20 nm (PEDOT:PSS), $\mathrm{LT} 3=11 \mathrm{~nm}(\mathrm{CuPC}), \mathrm{LT} 5=4 \mathrm{~nm}(\mathrm{PTCl})$ and LT5=12 nm (BCP). The incident light is normal to the structure (shown with arrows). For each architecture, only one unit cell of the repeating grating structure is visualized in the electric field profiles, using the same depicted color map for (a)-(c). The absorbing layer is also shown with the white lines.

To model plasmonic gratings integrated into thin-film organic solar cells, we used two-dimensional finite-difference timedomain simulator (developed by Lumerical Solutions, Inc., Canada). We analyzed three plasmonic architectures embedded into the same solar cell structure ITO/PEDOT:PSS/CuPc/PTCBl/BCP/Ag, as sketched in Fig. 1: for the case of only bottom silver grating (presented in Fig. 1(a)), the case of only top silver grating (presented in Fig. 1(b)), and the case of volumetric 
plasmonic resonators that includes both the top and bottom silver gratings (presented in Fig. 1(c)), all compared to the same negative control group of the non-metallic solar cell structure (with no silver grating). The solar cell included the layers of ITO with a thickness of LT1 $=150 \mathrm{~nm}$, PEDOT:PSS with LT2 $=20 \mathrm{~nm}$, CuPC with LT3 $=11 \mathrm{~nm}$, PTCl with LT5=4 nm and BCP with LT5=12 nm. We simulated all these architectures separately under normally-incident planewave illumination in both of TM polarization - with the magnetic field pointed along the $\mathrm{z}$-axis while the electric field is directed along $\mathrm{x}$-axis and TE polarization - with the electric field pointed along the $\mathrm{z}$-axis while the magnetic field is directed along $\mathrm{x}$-axis - . For plasmonic designs, a parametric study is carried out. Fig. 1 shows the electric field maps for the period of the grating $\mathrm{P}=200$ $\mathrm{nm}$, width of the top metal grating $\mathrm{w} 1=50 \mathrm{~nm}$, and width of the top metal grating $\mathrm{w} 2=30 \mathrm{~nm}$. The absorption spectrum is computed in the active layers of copper phthalocyanine $(\mathrm{CuPc})$ and perylene tetracarboxylic bisbenzimidazole $(\mathrm{PTCBl})$. The enhancement in total absorption $\left(\mathrm{A}_{\mathrm{TM}}\right.$ and $\left.\mathrm{A}_{\mathrm{TE}}\right)$ is then calculated for each of the three plasmonic architectures with respect to the negative control group under the illumination of TM- and TE-polarized light; the overall enhancement is then given by $\left(\mathrm{A}_{\mathrm{TE}}+\mathrm{A}_{\mathrm{TM}}\right) / 2$ (Fig. 2).
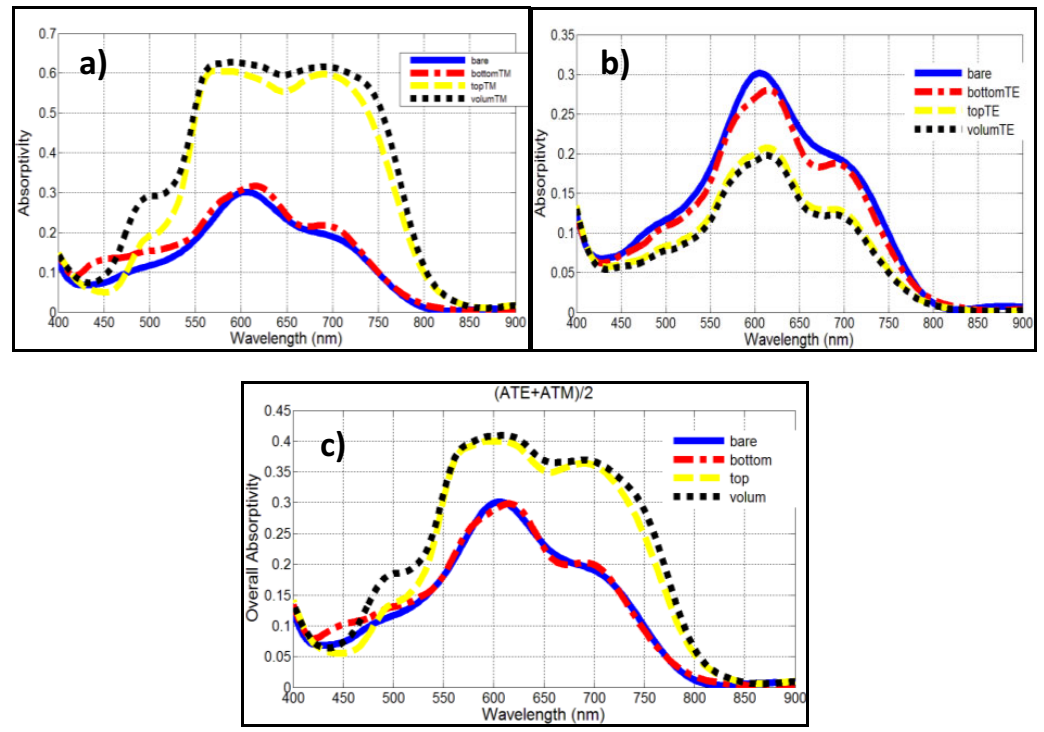

Fig. 2: Absorption spectra for the cases of three plasmonic architures (only top, only bottom and volumetric) under TM- and TE-polarized plane wave, compared with the negative control group (bare solar cell).

In our analysis, we observed that the absorbance is dominated by the CuPc layer in our structure (since this layer is thicker than PTCBl layer and the fields are more localized in this layer). For the first case of only top silver grating structure embedded in PEDOT:PSS layer partially, it is possible to obtain strong localized surface plasmon modes in a broad-band spectral range - from 450 to $850 \mathrm{~nm}$ - under the illumination of TM-polarized light. On the other hand, under TE-polarized light, the absorption spectrum is decreased. This behavior stems for the facts that the coupling of incoming light into the structure is blocked and the thin active layers do not allow TE waveguide modes. The optical absorption performance of the second architecture that consist of only bottom silver grating embedded into BCP layer partially shows a performance level very similar to that of the negative control group, bare solar cell. The weak surface plasmon modes generated around the metal gratings in this case, however, does not contribute to the absorption enhancement under the TE-polarized illumination. In TM polarization, the weak suppression in the absorption spectra is due to reflection from the bottom metallic grating. Our volumetric plasmon resonator architecture based on coupling two plasmonic gratings vertically also results in a great enhancement in the optical absorption under the TM-polarized illumination, slightly better than the first case of only top grating especially at the tails of the absorptivity spectrum. The surface plasmons generated by these metallic resonators exhibits greater electric field localization between these structures.

In summary, we proposed and demonstrated a volumetric plasmonic resonator architecture that embeds two vertically coupled plasmonic gratings in a single thin-film organic solar cell. Using the same film thicknesses, with this volumetric resonator, we showed an overall absorption enhancement $\left(\mathrm{A}_{\mathrm{TE}}+\mathrm{A}_{\mathrm{TM}}\right) / 2$ in the active layers of solar cells of $\sim 67 \%$ under the AM1.5G solar illumination. This novel architecture can be easily extended and applied to different types and materials of solar cells.

Acknowledgements: This work is supported by NRF-RF 2009-09, EU-FP7 Nanophotonics4Energy NoE, and TUBITAK EEEAG 107E088, 109E002, 109E004, and 110E010. HVD acknowledges support from ESF-EURYI and TUBA-GEBIP.

References

[1] H.A. Atwater, et al., Nature Materials, 9, 2629 (2010).

[2] M.L. Brongersma, et al., Nature Materials, 9, 2630 (2010).

[3] C. Min, et.al., Appl. Phys. Lett., 96, 133302 (2010). 